\title{
Intensity-volume interaction effects in gustatory perception'
}

\author{
R, A. M. GREGSON ANO G, L, PARIS \\ UNIVERSITY OF CANTERBURY, NEW ZEALAND
}

Three paired comparison type experiments, with 20 different $S s$ in each, using citric acid at three concentration levels as taste stimulus, investigated some effects of differences in stimulus liquid bulk on perceived relative taste intensity, and effects of concentration differences on perceived relative volume. Perceived relative taste intensity increases slightly with increasing liquid bulk and relative perceived bulk increases slightly with concentration, both effects being significant: $p<.01$. An explanation in terms of a taste-quantity constancy mechanism is advanced.

Any one dimension of variation of sensory input can in theory serve as the basis of cues to the presence of variation on another copresented dimension. Dimensional interaction of such a form has been explored widely within and between various sensory modalities (Epstein, Park, \& Casey, 1961; Gogel, 1963; Day, 1964; Kravkov, 1966). In vision the sizedistance constancies constitute both a special case of dimensional interaction and a paradigm for theories of perception which may subsequently be generalized to other modalities. There would appear to have been, as yet, no attempts to demonstrate an analogue for constancy phenomena in gustation, even though the possibility has been discussed (Osgood, 1953, p. 276). Constancies have been interpreted to mean that the perception of one sensory dimension is in part the consequence of collateral sensory input from other dimensions, and that this latter collateral information is used, centrally, by the organism to aid it in giving an invariant response to what is physically an invariant distal stimulus magnitude experienced under very varied circumstances (Brunswik, 1944).

The general multivariate situation can be considered in gustatory terms: By virtue of the physics of sensation a taste cannot be experienced without collateral stimulation from inter alia volume, weight, temperature, or viscosity (Gregson \& Matterson, 1967). This other stimulation may facllitate or perturb the perception of taste quality or intensity, and such interaction has to be quantitatively explored before it can be stated if taste constancy exists. This study examines interactions between the bulk of taste stimull, which necessarily involved both volume and weight, and their concentration perceived as intensity.

\section{EXPERIMENT I}

\section{Subjects}

Twenty persons from the university campus, 13 male and seven female, mainly first year psychology students, served as Ss. Ss' mean age was 21.5 years.

\section{Stimuli}

Citric acid at three concentrations $\left(C_{1}=.001 \mathrm{~g} / \mathrm{ml}\right.$, $\mathrm{C}_{2}=.002 \mathrm{~g} / \mathrm{ml}, \mathrm{C}_{3}=.0035 \mathrm{~g} / \mathrm{ml}$ ), all in deionized (equivalent. to triple distilled) water with resistance not less than $2 \mathrm{M} / \mathrm{cm}$, was run from burettes into $50 \mathrm{ml}$ beakers. Samples were in three volumes $\left(V_{1}\right.$ $=8 \mathrm{ml}, \mathrm{V}_{2}=16 \mathrm{ml}, \mathrm{V}_{3}=24 \mathrm{ml}$ ), and were kept in Gallenkamp constant temperature baths set at $25^{\circ} \mathrm{C}$. Ambient laboratory temperature was maintained in the range $17-22^{\circ} \mathrm{C}$.

\section{Procedure}

Ss sequentially tasted pairs of samples which either: (1) had the same concentration and volume or (2) had the same concentration but differing volumes, and on all pairs judged the taste intensity of the second stimulus in the pair relative to the first by selecting one from a symmetrical bipolar set of eleven statements running from "much more intense" through "equally intense" to "much less intense." These descriptive response statements were given Likert values 1 through 11 , respectively, but these numbers were never shown to Ss.

Each $S$ made 21 equal concentration paired comparisons, three equal volume comparisons and four unequal volume comparisons balanced for order at each concentration level. All Ss did the same 21 comparisons, but each $S$ did them in a different random order. Tastings were to a fixed time schedule signalled to $\mathbf{S}$ by Illuminated arrows. Each stimulus was held in the mouth for $5 \mathrm{sec}$, and $6 \mathrm{sec}$ elapsed between the stimuli within each pair. Ss had water rinse ad lib between paired comparisons. The experiment was conducted individually in the Gustatory Psychophysics Laboratory at the University of Canterbury. $E$ was not visible to $S$ during most of the experiment, and verbal communication was kept to a minimum.

\section{Results}

The mean scaled relative intensities pooled over all $20 \mathrm{Ss}$ for each of the 21 paired comparisons are given in Table 1.

A value of 6 corresponds to equality of perceived taste intensity; values below 6 imply that the second stimulus was more intense, and vice versa for values above 6. In the case of the comparisons $v_{1}-v_{1}, v_{2}-V_{2}$, and $V_{3}-V_{3}$ the difference between the observed mean score and a score of 6 gives a measure of time-order error for taste intensity. This time-order error decreased as concentration increased, becoming nega- 
Table 1.

Mean Relative intensity ratings of comparisons in Experiment 1.

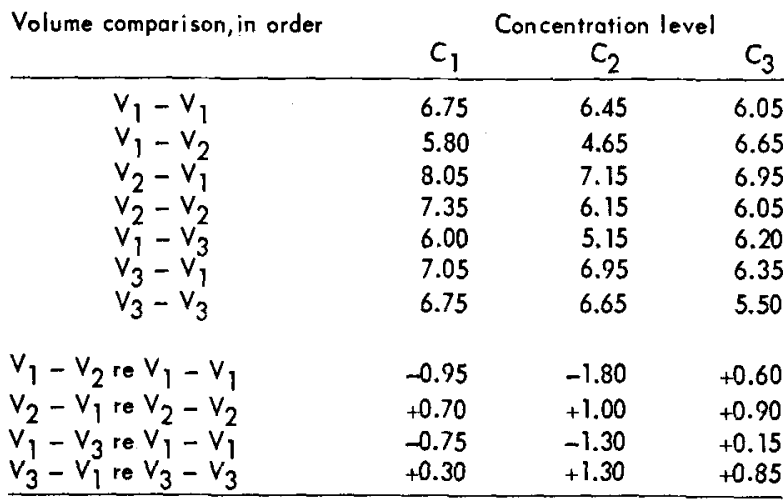

tive for $\mathrm{C}_{2} \mathrm{~V}_{3}-\mathrm{C}_{2} \mathrm{~V}_{3}$ and $\mathrm{C}_{3} \mathrm{~V}_{3}-\mathrm{C}_{3} \mathrm{~V}_{3}$. This concentration effect is significant $(F=3.88, \mathrm{df}=2 / 171, p<.025)$; a volume effect on the error is apparently present but too slight to achieve significance.

Given a sequential comparison of the general form: "Taste $C_{i} V_{i}$, then taste $C_{i} V_{j}$, and compare apparent concentrations," the corresponding control for timeorder error alone, without a differential volume effect, is: "Taste $C_{i} V_{i}$, then taste $C_{i} V_{i}$ again." Assuming that the time-order and volume effects summate linearly (Gregson, 1964a, b), the difference of the ratings on the two paradigm pairs above is a firstorder approximation to the relative volume effect at the levels $C_{i}, V_{i}, V_{j}$. Figure 1 is derived by making the appropriate subtractions in the upper values in Table 1, and thus gives net effects rather than absolute ratings. Positive deviations represent comparisons where the intensity of the first stimulus is perceived to be greater than that of the second, which in most cases are also those comparisons in which the first stimulus is greater in volume than the second in a pair. The exceptions are at $C_{3}$. Negative deviations represent comparisons in which the intensity of the second stimulus is perceived to be greater than that of the first, which are all comparisons in which the second stimulus has the greater volume.

In the case of the unequal volume comparisons the effect of tasting the larger stimulus before the smaller, as compared with the other way around, is marked; the direction main effect has $F=20.0$ (df= $1 / 228, p<.01)$. The direction by concentration interaction was also significant $(F=3.7, d f=2 / 228, p<.025)$. This is reflected in Fig. 1 in the funnelling tendency, at $\mathrm{C}_{3}$, of the trend lines representing the four comparison conditions.

A taste intensity-volume interaction effect is seen to exist, whereby volume differences cause a slight breakdown in intensity constancy.

\section{EXPERIMENT 2}

Having shown that bulk, i.e., volume or weight, differences perturb small relative intensity judgments, the converse situation in which concentration differences could modify relative volume judgments was next explored, using a precisely similar paradigm.

\section{Subjects}

Twenty persons from the university campus, 10 male and 10 female, with a mean age of 20.5 years, served as Ss. None had served in Experiment 1.

\section{Stimuli}

The same intensity and volume levels were employed as in Experiment 1, except that unequal pairs were now unequal in concentration but equal in volume, and relative volume responses rather than

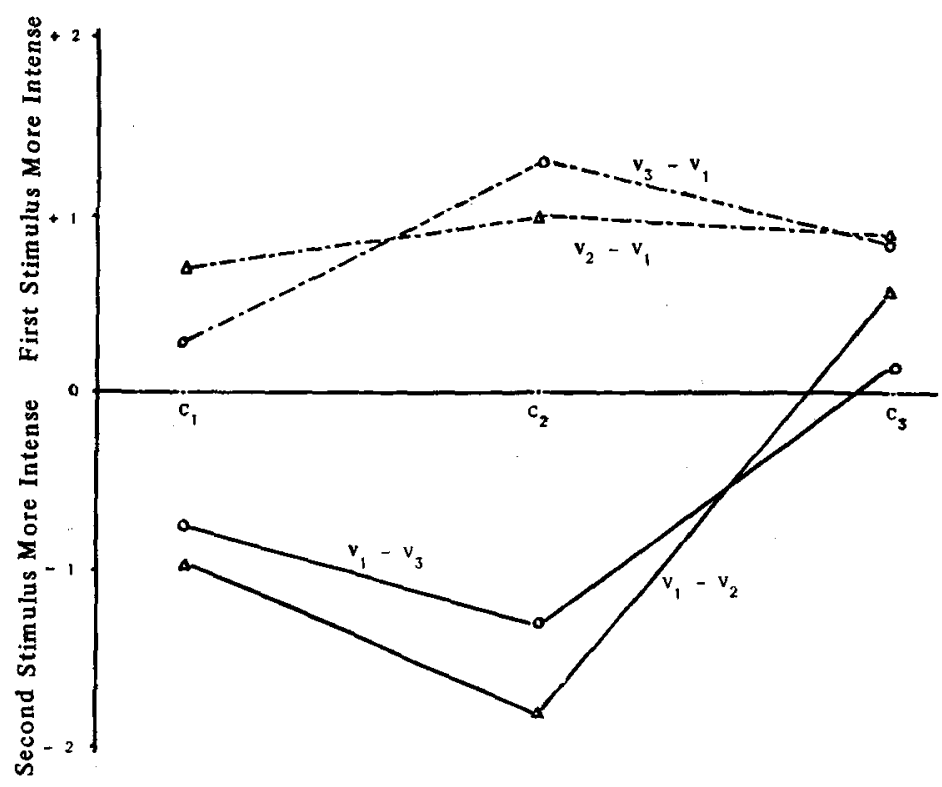

Fig. 1. Scaled extent of perce ived inequalities in intensity of the equal-concentration unequal-volume paired comparisons in Experiment 1. 
relative intensity responses were elicited on a set of 11 statements about volumes, scored in the same manner as for Experiment 1.

\section{Procedure}

Each $\mathbf{S}$ made 21 equal volume paired comparisons, three equal concentration comparisons and four unequal concentration comparisons; all other details were comparable to Experiment 1.

\section{Results}

The results followed very closely the pattern of Experiment 1, so only the graph of net deviations, Fig. 2, is reproduced. The time-order effects were about the same scaled magnitudes as in Experiment 1 and varied as a function of volume significant at $F=5.250 \quad(\mathrm{df}=2 / 171, \mathrm{p}<.01)$. The effect of tasting the solution of greater concentration before the lesser (both having the same volume) as compared with the other way around is marked; the direction main effect has $F=34.517 \quad(\mathrm{df}=1 / 228, p<.001)$. A direction by concentration interaction was also significant $(F=6.184$, $\mathrm{df}=1 / 228, \mathrm{p}<.025)$; there is again a funnelling trend in the graph, this time at $V_{3}$. The positive directions represent comparisons where S's net judgment makes the first stimulus perceptibly greater in volume, and are all comparisuns in which the second stimulus is less concentrated. The negative deviations represent comparisons where $\mathrm{S}$ perceives the second stimulus as greater in volume, which in all but one case are pairs in which the second stimulus is more concentrated. Again, a taste intensity-volume interaction is found, in this case concentration differences cause a slight breakdown in volume constancy, in an analogous sense to the corresponding breakdown in intensity constancy of the previous experiment.

\section{Method}

\section{EXPERIMENT 3}

It may cogently be argued that the magnitude and direction of the effects reported in Experiment 1 were necessarily modified by the fact that Ss could see the volume differences involved, even though the instructions demanded only relative intensity judgments. Accordingly, a third experiment which exactly replicated Experiment 1, except in that the $50 \mathrm{ml}$ beakers used were coated with opaque black, was conducted. This change reduced the role of consistent or facile visual cues in the task.

\section{Subjects}

Ss were 20 persons from the university campus, 10 male and 10 female, with a mean age of 20.2 years. None had served in either of the previous experiments.

\section{Results}

The data obtained in Experiment 3 were treated in exactly the same way as previously. Only the graph of net effects is reproduced here, in Fig. 3. The time-order errors were again of comparable magnitude, and varied as a function of concentration ( $F$ $=6.091, \mathrm{df}=2 / 171, \mathrm{p}<.001)$ and of volume $(F=5.019$, df $=2 / 171, p<.001$ ).

As in Experiment 1 , the consequence of tasting the larger stimulus before the smaller as compared with the other way around is marked ( $F=33.602$, $\mathrm{df}=1 / 228, \mathrm{p}<.001)$. The direction by concentration interaction is also significant $(F=3.631, \mathrm{df}=2 / 228$, $\mathrm{p}<.05$ ).

Figure 3 is by no means as clear as Fig. 1, but exhibits comparable trends: Whether or not visual cues are reduced, in the sense of a visual "reduc-

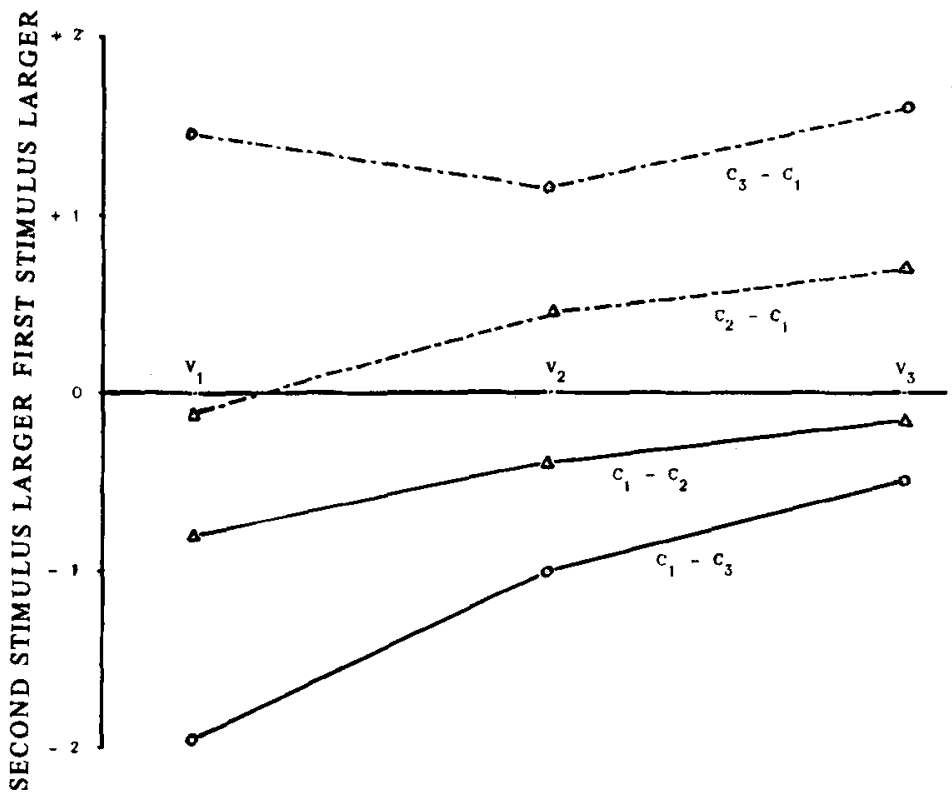

Fig. 2. Scaled extent of perceived unequalities in volume of the equal-volume unequalconcentration paired comparisons in Experiment 2. 


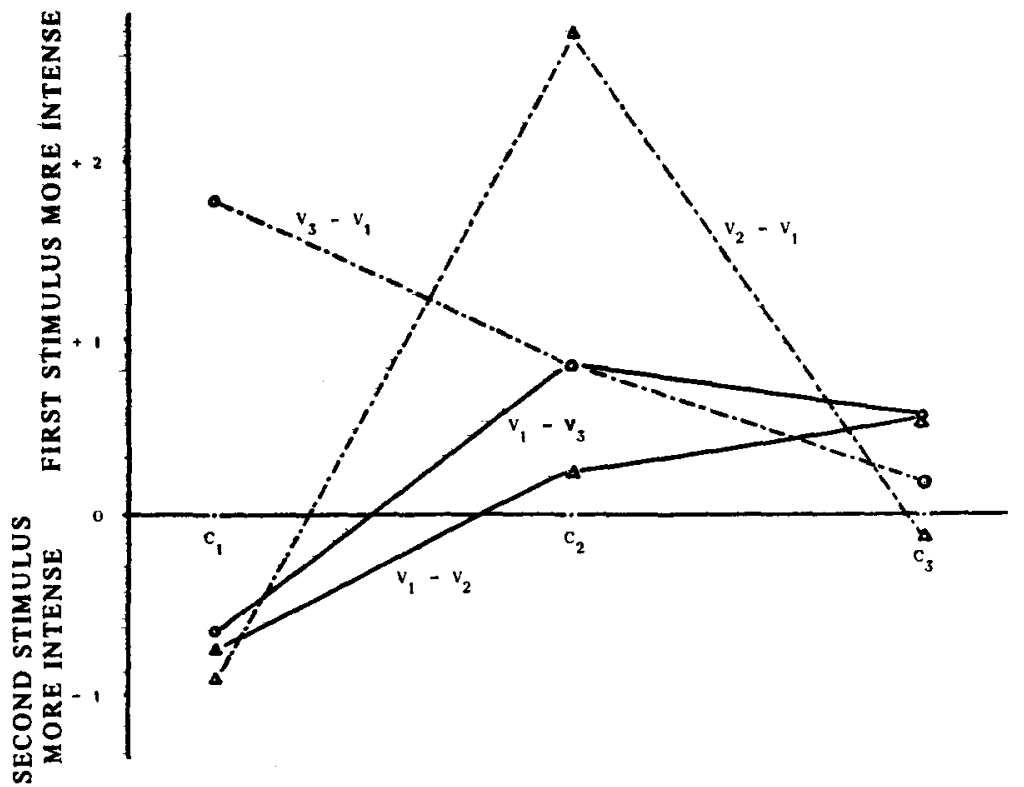

Fig. 3. Scaled extent of perceived inequalities in intensity of the equal-concentration unequal volume paired comparisons with visual cues restricted in Experiment 3. tion screen" experiment, the dimension of liquid bulk serves as a slightly misleading cue to perceived relative taste concentration.

\section{DISCUSSIOH}

The symmetry of the results obtained rules out a simple physiological explanation at the receptor level; while it is possible to explain the effect of volume on perceived relative intensity as being consistent with some gustatory receptor systems functioning in series rather than in parallel, the obtained effect of intensity on volume does not simply follow-more postulates about receptor level mechanisms would be needed. There are, in any case, no good grounds for deriving explanations of gross gustatory psychophysical phenomena from detailed receptor physiology (Beidler, 1962).

The phenomena reported here are more readily considered as examples of centrally-mediated judgment processes. In effect, $S$ is presented with three dimensions in the first member of each pair, taste intensity, tasteless volume or weight, and taste amount which is an integral of intensity over the volume. When the second stimulus is to be compared, even with one of these three dimensions fixed, the other two must vary if the second stimulus differs on one of either concentration or volume. For example, if volume is fixed, than an increase in concentration produces a corresponding increase in taste amount as well as in intensity. The increase in concentration may be perceived as an intensity change, but if an intensity response is excluded and a volume response asked for, this constraint induces a non-zero subjective probability in $S$ that a volume difference exists; then the consequent shift in taste amount is instead interpreted by $S$ as a change due to a volume increase. This argument still fits if the roles of concentration and volume are reversed, because each has the same relation to taste amount.

In most taste experience, if stimuli are manipulated then the most ready operation is dilution to a hedonically optimum concentration. This operation leaves taste amount approximately constant, ignoring threshold corrections, so an expectation for an inverse relation between volume and intensity, and an independence of taste quantity from either volume or concentration, are part of adult experience. In the paradigms used here these expectations are not met, as taste quantity increases with volume or with concentration. Intensity and volume constancies break down, to a small but statistically significant degree, and this is postulated to occur because the organism tries to preserve a taste quantity constancy. The latter constancy breaks down in favor of volume or intensity constancies as soon as the magnitudes of these two sensory dimensions are large, because the amount of stimulation then involved is sufficient to swamp the perceptual noise in the task which permits the quantity constancy to operate to yield nonveridical perception. This breakdown in quantity constancy explains the tendency for all the graphs presented to show funnelling at the right hand side. It remains to be demonstrated whether or not these postulated gustatory constancies are or are not proper analogues of any visual constancies.

\section{References}

Beidler, L. M. Taste receptor stimulation. Prog. Biophys. Biophys. Chem., 1962, 12, 109-151.

Brunswik, E. Distal focussing of perception: size constancy in a representative sample of situations. Psychol. Monogr.. 1944, 56, No. 1 (Whole No. 254).

Day, R. H. Perception. Annu. Rev. Psychol., 1964, 56, 1-28. 
Epstein, W., Park, J., \& Casey, A. The current status of the sizedistance hypothesis. Psychol. Bull., 1961, 58, 491-514.

Gogel, W. C. The visual perception of size and distance. Vision Res., 1963, 3, 101-120.

Gregson, R. A. M. Fitting a linear trace decay model to taste comparisons and preferences. Brit. J. Statist. Psychol., 1964a, 17, 137-151.

Gregson, R. A. M. Modification of percelved relative intensities of acid tastes by ambient illumination changes. Austral. J. Psychol., 1964b, 16, 190-199.

Gregson, R. A. M., \& Matterson, Helen M. Psychophysical parameters of the perception of orally-retained liquid bulk. Percept. \& Psychophys., 1967, 2, 89-90.
Kravkov, S. V. Interaction of the sense organs. In Psychol. Res. in the U.S.S.R., 1, Moscow: Progress Publishers, 1966. Pp. 218266.

Osgood, C. E. Method and theory in experimental psycholooy. New York: Oxford University Press, 1953.

\section{Note}

1. Data reported here were collected by Mr. G. L. Paris in part fulfilment of $M$.A. degree requirements. The research was supported by funds from the U.G.C. of New Zealand.

(Accepted for publication May 30, 1967.) 